\title{
13. An irradiation facility with a horizontal beam for radiobiological studies
}

\author{
J.Czub ${ }^{1}$, T. Adamus ${ }^{5}$, D. Banaś ${ }^{1,2}$, J. Braziewicz ${ }^{1,2}$, J. Choiński, J. Dyczewski ${ }^{4}$, U. Górak ${ }^{4}$, M. \\ Jaskóła ${ }^{3}$, A.Korman ${ }^{3}$, K. Kuchta ${ }^{4}$, Z. Szefliński ${ }^{4}$, A.Wójcik ${ }^{5,6}$ \\ 1) Institute of Physics, Świętokrzyska Academy, ul.Świętokrzyska 15, 25-406 Kielce, \\ 2) Holycross Cancer Center, ul. Artwińskiego 5, 25-389 Kielce, \\ 3) The Andrzej Soltan Institute for Nuclear Studies, 05-400 Otwock-Swierk, \\ 4) Institute of Experimental Physics, Warsaw University, ul. Hoża 69, 00-681 Warsaw, \\ 5) Institute of Biology, Świętokrzyska Academy, ul. Świętokrzyska 15, 25-406 Kielce, \\ 6) Institute of Nuclear Chemistry and Technology, ul. Dorodna 16, 03-195 Warsaw,
}

A facility with a horizontal beam for radiobiological experiments with heavy ions has been designed and constructed at the Heavy Ion Laboratory in Warsaw University. The facility is 
optimal to investigate the radiobiological effects of charged heavy particles on a cellular or molecular level as the plateau of the Bragg curve as well as in the Bragg peak. The passive beam spread out by a thin scattering foil provides a homogeneous irradiation field over an area of at least $1 \mathrm{x} 1 \mathrm{~cm}^{2}$. For in vitro irradiation of biological samples the passive beam spreading combined with the $x-y$ mechanical scanning of the irradiated sample was found to be an optimum solution. Using $x-y$ step motor, the homogenous beam of ions with the energy loss range in the cells varied from $1 \mathrm{MeV} / \mu \mathrm{m}$ to $200 \mathrm{keV} / \mu \mathrm{m}$ is able to cover a $6 \mathrm{~cm}$ in diameter Petri dish that holds the biological samples [1]. Moreover on-line fluence monitoring based on single-particle counting is performed to determine the dose absorbed by cells.

\section{Introduction}

The use of heavy ion accelerators has enjoyed extensive interest for biophysical experiments over the past years. The radiobiological effects of charged heavy particles on a cellular or molecular level are of fundamental importance in the field of biomedical applications, especially in hadron therapy and space radiation biology.

The study of the influence of high linear energy transfer (LET) ions on the DNA in the cell nucleus yields a basic information to the models describing the effects of radiation of different cells, the track structure of projectiles passing in the cell and the DNA geometry [2]. The use of heavy ions in radiotherapy ("hadrontherapy") could result in effective treatment due to a very good depth dose profile ("Bragg curve") and the high relative biological effectiveness (RBE) for cell inactivation. Nowadays, there are some clinical centers and research facilities performing treatments with protons and heavy ions. Therefore the study of biological characteristics of different particles has to be known to avoid therapy-induced side effects. The knowledge of radiation-induced DNA-breaks is also the base to estimate the risk from radiation exposure during long-manned space flights. There is also increasing interest in learning the influence of ionizing particles on the apoptosis or cell membrane damage.

The main aim of the work was to set up a facility for radiobiological experiments for different ionizing particles in the region of the Bragg peak. The special attention has been concentrated on the information concerning the effectiveness of the low-energy ions, where the dosimetry of slow particles around the Bragg peak is a technical challenge. The V79 Chinese hamster cells were used for the first radiobiological experiments with carbon ions.

\section{Technique}

\subsection{Irradiation facility}

At the designing process we have made the cardinal assumption that the facility will be used for irradiation of biological samples under physiological conditions by various ions at a wide range of LET. The U-200P cyclotron of the Heavy Ion Laboratory provides heavy ions from boron up to argon with energies from $2 \mathrm{MeV} / \mathrm{amu}$ to $10 \mathrm{MeV} / \mathrm{amu}$. At this facility almost all types of ions, which are potentially useful in hadrontherapy can be accelerated, so different radiobiological experiments covering a wide range of LET values are possible. The set-up with a horizontal beam has been constructed.

The ion beams are transported from the cyclotron area to the set-up by a horizontal beam line [3] with a conventional beam tuning components including a fast mechanical beam shutter, which can be activated by the irradiation control system. Starting from the switching magnet of the cyclotron the beam line consists of the following components:

- pneumatic operated valve, 
- turbo molecular pumping system,

- scattering chamber with collimating system, target holder and detectors,

- beam tube (about $2.3 \mathrm{~m}$ long) with vacuum gauge

- pressure pick-up activating safety valve

- exit Havar window

- irradiation system mounted at the X-Y sliding table

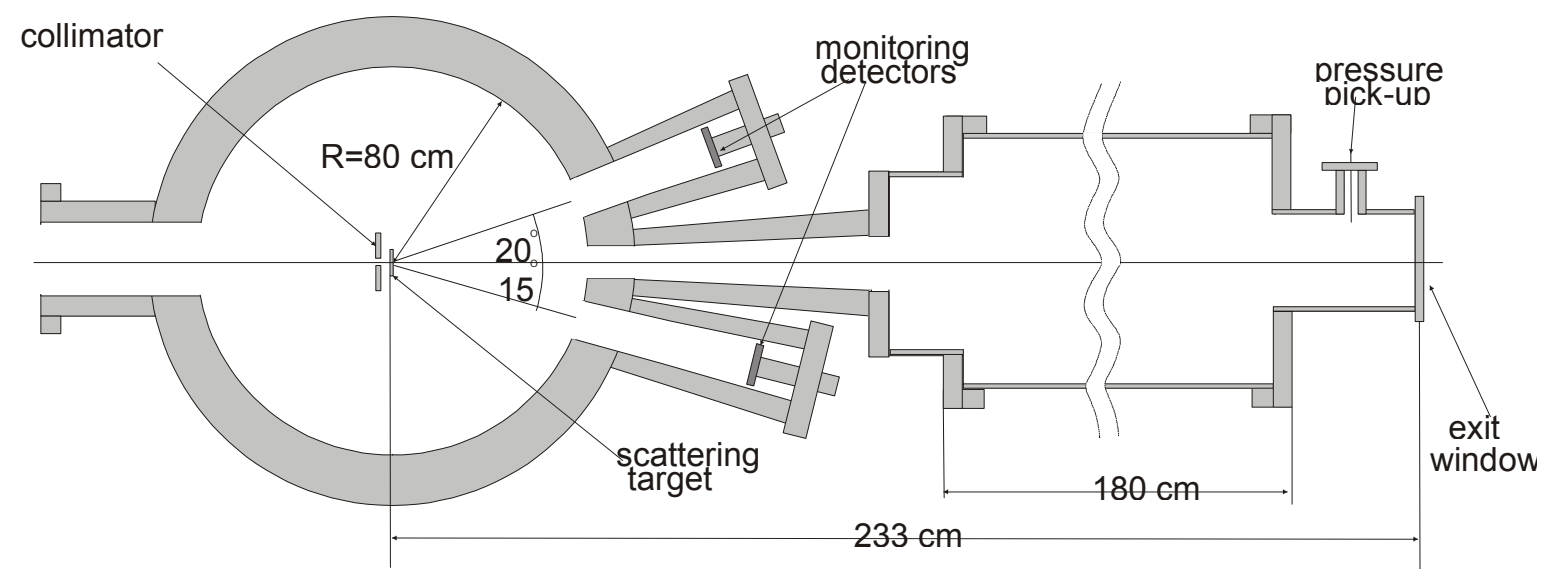

Fig. 1. The scheme of the set-up for radiobiological studies with the horizontal beam line at the U-200P cyclotron of the Heavy Ion Laboratory.

A protective failure system has been interlocked into the vacuum gauge circuit. This failure system shuts of the pneumatically opened safety-valve at the pressure of $10-4 \mathrm{~mm} \mathrm{Hg}$. At a pressure greater than 10-1 $\mathrm{mm} \mathrm{Hg}$ the vacuum pumps are shut down also. At the entrance into the radiobiological set-up (see figure 1) the beam is collimated by a $2 \mathrm{~mm}$ in diameter aperture.

To achieve a homogeneous radiation field over the area of the exit window the beam is passively spread out by scattering foil of high atomic number material with appropriate thickness. Usually, gold scattering foils in the $3-50 \mathrm{mg} \cdot \mathrm{cm}^{-2}$ thickness range are used. The ion beam intensity is analyzed on-line by an integral monitoring system of two silicon detectors placed inside the scattering chamber at the angles of $15^{\circ}$ and $20^{\circ}$. The irradiation ion beam parameters are also examined at separate measurements by a removable diagnosis system following the exit window.

The beam extraction in air is performed through a quadratic window with $10 \mathrm{~mm}$ side length sealed by a Havar foil with $2.45 \mathrm{mg} \cdot \mathrm{cm}^{-2}$ thickness. The foil is cemented to stainless-steel flange using epoxy glue. The thickness of this foil prevents the break of the window due to the pressure gradient.

\subsection{Beam monitoring and dosimetry}

Two independent detector systems for the beam diagnosis and for the beam monitoring were used: the Si-detector measuring the particle flux at the position of the cell container and two 
on-line detectors monitoring the particle flux by intensity of the scattered particles. The intensity and the distribution of the scattered ions was measured outside of the exit window (at the position of the Petri dish holding the cells) by surface-barrier silicon detector fastened to a $\mathrm{x}-\mathrm{y}-\mathrm{z}$ sliding table and moving across the two dimensional array centers, distant each of other by $0.5 \mathrm{~mm}$. To reduce the count rate and to obtain information about particle flux from the fine window area an Al collimator with opening diameter of $0.5 \mathrm{~mm}$ was placed in front of the detector. The fast electronic circuit of the charge sensitive pre-amplifier allows rates up to $10^{3}$ particles/s without intolerable losses.
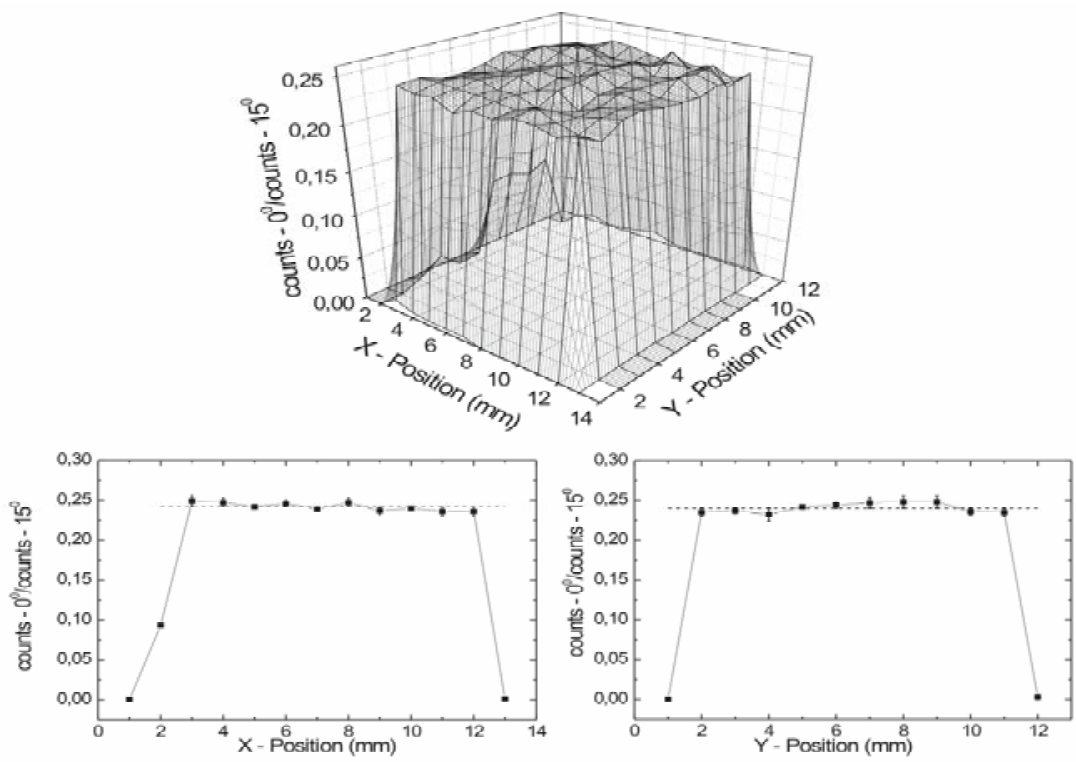

Fig. 2. Measured two dimensional plot of the ${ }^{12} \mathrm{C}$ ions intensity scattered over the $1 \times 1 \mathrm{~cm}^{-2}$ exit window at the cell container position normalized to the intensity of the elastically scattered ions at the $15^{\circ}$ silicon detector (a) and the profiles in $\mathrm{x}-(\mathrm{b})$ and $\mathrm{y}$-direction (c).

The detectors mounted stationary in the scattering chamber at the angle of $15^{\circ}$ and $20^{\circ}$ measure on-line the intensity of the scattered ions at the scattering foil during cell irradiation. To reduce the count rate, collimators with $2 \mathrm{~mm}$ diameter were placed in front of each detector. The $20^{\circ}$ detector was placed at a distance of $118 \mathrm{~mm}$ from the center of a scattering foil and the $15^{\circ}$ detector at $147 \mathrm{~mm}$. The typical spatial intensity distribution of the ${ }^{12} \mathrm{C}$ scattered ions over exit window, is presented in figure 2. The distribution was measured by silicon detector in air at the biological sample position and normalized to the yield of on-line scattered particles at the $15^{\circ}$. Combining scattering of the beam with mechanical displacement of the detector, the beam uniformity of $2.5 \%$ over area of $6 \times 6 \mathrm{~cm}^{2}$ was obtained.

Our irradiation facility allows to vary the available energy for radiobiological experiments in the range of $\sim 2 \mathrm{MeV} / \mathrm{amu}$ to $\sim 10 \mathrm{MeV} / \mathrm{amu}$. At the energy of $\sim 2 \mathrm{MeV} / \mathrm{amu}$ the energy loss in the biological cells is about $1 \mathrm{MeV} \cdot \mu^{-1}$ and the range of ions in water $\sim 10 \mu \mathrm{m}$ which correspond to a typical cell diameter, while at the energy of $\sim 10 \mathrm{MeV} / \mathrm{amu}$ the energy loss reaches the value of $200 \mathrm{keV} \cdot \mu \mathrm{m}^{-1}$ and the range of $\sim 400$ micrometers. 


\subsection{Data readout and visualization}

In radiobiological experiments with charged particles the cells are typically seeded at the Petri dishes with a typical diameter of 5-6 cm which let to minimize the errors concerning the statistics of cells and events. At our facility the biological cells should be seeded at the Petri dishes as a plastic ring with $4.8 \mathrm{~cm}$ diameter glued to the bottom of a thin $3 \mu \mathrm{m}$ Mylar foil. A special construction of the top of this Petri dish allows also for cell irradiation under physiological conditions.

To obtain homogeneous irradiation of all the cells the Petri dishes were placed vertically in a specially designed sample holder mounted into the controlled $x-y-z$ stepping motor with remote control. At this system time dependence of the beam intensity as well as the energy spectrum of the scattered beam is registered. The signals from the monitor detectors mounted in the vacuum are counted in the fast programmable scaler. When the number of registered particles reaches the preset value (characteristic for settled dose), a start signal is created and the target changes its position according planned route. During irradiation all count rates are measured with a sampling rate of $100 \mathrm{~Hz}$ and stored on hard disc. The data are on-line visualized at the PC's monitor by a graphical interface. The communication between the PC's and electronics takes place with CAMAC crate controller and located in control system. From these data an off-line dose-analysis can be performed [1].

When the beam is stopped, the count rate is reduced to zero. The next sample is turned into the irradiation position and the irradiation resumes with the next characteristic presume rate. From the plot it is also evident that the accelerator beam stability does not play important role in the irradiation process. The exit window and sliding table with the samples to be irradiated are controlled by a video camera.

\section{Conclusions}

The radiobiological facility at the Heavy Ion laboratory is operational. The facility allows to vary the available energy for radiobiological experiments in the range of $\sim 2 \mathrm{MeV} / \mathrm{amu}$ to $\sim 10$ $\mathrm{MeV} / \mathrm{amu}$. At the energy of $\sim 2 \mathrm{MeV} / \mathrm{amu}$ the energy loss in the biological cells is about 1 $\mathrm{MeV} \cdot \mu \mathrm{m}^{-1}$ and the range of ions in water $\sim 10 \mu \mathrm{m}$ which correspond to a typical cell diameter, while at the energy of $\sim 10 \mathrm{MeV} / \mathrm{amu}$ the energy loss reaches value of $200 \mathrm{keV} \cdot \mu \mathrm{m}^{-1}$ and range of $\sim 400$ micrometers. Lastly, some experiments were done to test the new setup and the results show that the system works in a satisfactory manner. A biological program with experiments studying the relative biological efectiveness and chromosomal aberrations will be started soon.

The work was partially supported by the Polish State Committee for Scientific Research under Grant No. 1P03B10527.

\section{References}

[1] J.Czub, T.Adamus, D.Banaś, J.Braziewicz, J.Choiński, J.Dyczewski, M.Jaskóła, A.Korman, Z.Szefliński, A.Wójcik, Warsaw University, Heavy Ion Laboratory, Annual Report (2004) p. 38-41

[2] Kraft, G. Prog. Tumor therapy with heavy charged particles. Part. Nucl. Phys, 45, 473544,(2000).

[3] Scampoli, P. and Casale, M. and Durante, M. and Grossi, G. and Pugliese, M. and Gialanella, G. Low-energy light ion irradiation beamline for radiobiological studies. Nucl. Instr. and Meth. B 17, 337-343 (2001). 